\title{
MODELLING OF ATMOSPHERIC FLOW AND DISPERSION IN THE WAKE OF A CYLINDRICAL
} OBSTACLE

\author{
S. Andronopoulos ${ }^{1, *}$, I. Mavroidis $^{2}$, A. Venetsanos $^{1}$, J.G. Bartzis ${ }^{3}$ \\ ${ }^{1}$ Environmental Research Laboratory/INT-RP, NCSR Demokritos, Aghia Paraskevi, Greece \\ ${ }^{2}$ Hellenic Open University, Patras, Greece \\ ${ }^{3}$ University of Western Macedonia, Dept. of Engineering and Management of Energy Resources, Kozani, Greece
}

\begin{abstract}
This paper presents computational simulations of atmospheric dispersion experiments conducted around isolated obstacles in the field. The computational tool used for the simulations was the code ADREA-HF, which was especially developed for the simulation of the dispersion of positively or negatively buoyant gases in complicated geometries. The field experiments simulated involve a single cylindrical obstacle normal to the mean wind direction and two upwind sources of ammonia and propane, with the ammonia source located at different lateral positions (Mavroidis et al., 2003). Concentrations and concentration fluctuations for both gases were calculated by the model and compared with the experimental results to evaluate the model performance. Specific characteristics of dispersion were investigated using the computational tool. Comparisons of experimental and model results with the case of dispersion around an isolated cubical obstacle are also presented and discussed.
\end{abstract}

Key words: atmospheric dispersion; circular cylinder; wake; model evaluation; concentration fluctuations; laterally displaced source.

\section{INTRODUCTION}

The interaction of continuous plumes released from point sources with buildings and other structures is the major factor affecting short-range dispersion of atmospheric pollutants in built-up areas. The interaction between a plume and an isolated simple structure is crucial for detecting and isolating the main characteristics of building influenced dispersion. Field trials and wind tunnel modelling studies are often used, in order to improve the understanding of the physical processes involved and provide the necessary information to develop and validate mathematical modelling approaches as a practical tool. Most of the research on building-influenced dispersion is concerned with the examination of flow and dispersion around rectangular obstacles. However, information on flow and dispersion around circular cylinders is also very useful, for example in evaluating doses due to releases of hazardous materials near industrial installations or storage facilities. The flow around a vertically mounted three dimensional cylinder has certain similarities with the flow around a rectangular obstacle normal to the flow, since a horseshoe vortex is similarly generated near the ground upwind of the cylinder. The flow separates at the top of the cylinder and usually reattaches on the rear of the roof, and separates again at the back of the cylinder reattaching further downwind. A pair of trailing vortices is also generated on the upwind edges of the cylinder's top. However, flows around rectangular and cylindrical obstacles display some differences, since the cylinder does not have sharp edges, and therefore separation at the sides is less clearly defined than for a rectangular obstacle.

The present work aims at calculating mean concentrations and concentration fluctuations downwind of a single cylindrical obstacle using the CFD code ADREA-HF, in order to: (a) evaluate the model performance by comparison with field experimental data, (b) examine specific characteristics of dispersion of a laterally displaced plume, (c) investigate the influence of factors such as turbulence modelling scheme or variability in the field experiments (d) compare the results with those of computational simulations carried out around an isolated cubical obstacle (Mavroidis et al., 2007). Results regarding the above issues are presented in the current short paper.

\section{METHODOLOGY}

\section{Field Experiments}

Field experiments, described in detail by Mavroidis et al. (2003), were conducted at an experimental site on the northwest coast of England, under neutral weather conditions (corresponding to Pasquill stability class D). The surrounding terrain was generally flat. In the trials simulated here a cylindrical model building with its height equal to its diameter was used $(\mathrm{H}=\mathrm{D}=1.15 \mathrm{~m})$. A dual source/receptor system technique was used involving the simultaneous release of two different tracer gases (ammonia and propane) from two point sources, and the deployment of two different types of co-located detectors (the flame ionization detectors (FIDs) and the ultra-violet ion collector (UVIC) detectors). The tracer gas was released at a constant rate from an open-ended pipe, upwind of the obstacle. The two sources were co-located at the beginning of each experimental session at a location $2.0 \mathrm{H}$ upwind of the centre of the front face of the obstacle and at a height of $0.5 \mathrm{H}$. Then the ammonia source was moved in steps laterally, away of the axis defined by the mean wind direction and the centre of the obstacle. Two pairs of co-located detectors were centrally located $0.5 \mathrm{H}$ and $3.0 \mathrm{H}$ downwind of the rear face of the obstacle, while two more UVICR detectors were located $2.0 \mathrm{H}$ and $5.0 \mathrm{H}$ downwind of the rear face of the obstacle. The tracer gas flowrate was low enough to ensure that source momentum effects were not significant. No buoyancy effects have been observed during the experiments. The experimental configuration, including the lateral source locations investigated, is shown in Figure 1.

In the present paper the five experimental cases listed in Table 1 have been computationally simulated. 


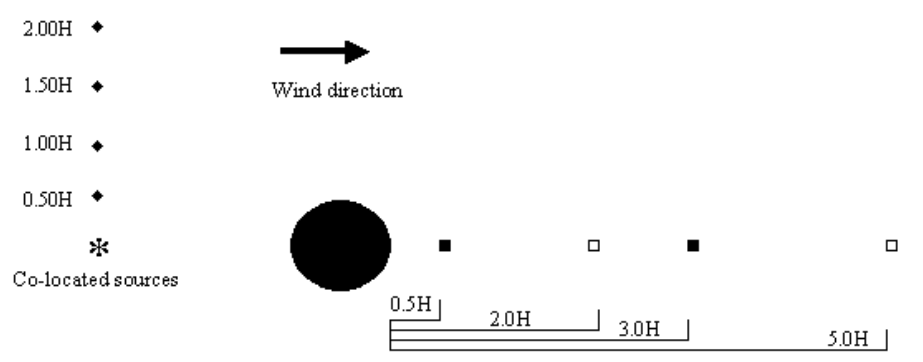

UVIC detector

- Co-located UVIC and FID detectors

- Ammonia source locations

Figure 1. Plan view of the experimental configuration, including all the lateral source locations investigated during the 5 experiments, as well as the sensors locations.

Table 1. Experimental characteristics for the five simulated experiments.

\begin{tabular}{|c|c|c|}
\hline $\begin{array}{c}\text { Experimental } \\
\text { case }\end{array}$ & $\begin{array}{c}\text { Lateral ammonia source } \\
\text { displacement }(\mathrm{xH})\end{array}$ & $\begin{array}{c}\text { Mean wind speed } \\
\left(\mathrm{ms}^{-1}\right)\end{array}$ \\
\hline $\mathrm{n} 01$ & 0.00 & 3.350 \\
\hline $\mathrm{n} 02$ & 0.50 & 2.647 \\
\hline $\mathrm{n} 05$ & 1.00 & 3.530 \\
\hline $\mathrm{n} 11$ & 1.50 & 4.457 \\
\hline $\mathrm{n} 07$ & 2.00 & 3.553 \\
\hline
\end{tabular}

\section{Computational tool and modeling approach}

The computational fluid dynamics code ADREA-HF, developed by the Environmental Research Laboratory, has been used for the simulations presented in this article. The purpose of ADREA-HF is to simulate the dispersion of buoyant or passive pollutants over complex geometries. ADREA-HF is a finite volumes code that solves the Reynolds-averaged equations for the mixture mass, momentum, energy, pollutant mass fraction and the variance of the pollutant mass fraction. Turbulence closure is obtained through the eddy viscosity concept, which is calculated either by a 1 -equation $k-1$ model or by the standard $k-\varepsilon$ model. The turbulent kinetic energy $k$ and the dissipation rate are calculated by transport equations. The effective length scale $l$ depends on the flow stability and on the distance from solid boundaries, so in the general case it is three-dimensional. For the pollutant concentration variance, a threedimensional transport equation is also solved. Details on the modelling approach regarding the concentration variance are included in Andronopoulos et al. (2002).

The experimental characteristics sources and physical properties of the two gases were used for the simulations. In accordance with the field experiments, the atmospheric stability conditions were taken as neutral for all modelled cases. The computed concentrations and concentration fluctuations were non-dimensionalised, to be directly comparable with the experimental data (Mavroidis et al., 2003). Two different simulations were performed for each experimental case listed in Table 1: one with the ammonia source located at the proper lateral position and one with the propane source which was always located on the centreline.

\section{RESULTS AND DISCUSSION}

Figure 2 shows contours of calculated ammonia mean concentration and concentration fluctuations for experimental cases N01 and N05. The contours are drawn at the horizontal plane located at the height of the gas source and the detectors. In case N01, a characteristic bifurcation of the plume in the near-wake of the obstacle, attributed to the capture of the plume by the horseshoe vortex generated upwind of the obstacle, is apparent both from the concentration and concentration fluctuation contours. In case N05 the ammonia source was displaced in the crosswind direction by $1.0 \mathrm{H}$. In this case it can be observed that the plume is entrained in the wake downwind of the obstacle and that concentration fluctuations are a little higher in magnitude than concentrations downwind of the building.

As already noted, the propane source was at the same location for all the cases listed in Table 1, while the atmospheric stability conditions were neutral in all cases and the wind speed and direction did not differ significantly between the cases. Therefore, the propane release was actually repeated 5 times, allowing an ensemble average to be obtained. The experimental and computational ensemble averages have been compared at the positions $0.5 \mathrm{H}$ and $3.0 \mathrm{H}$ downwind of the obstacle, where propane concentrations were measured in the field. This is an important type of model performance evaluation since Reynolds-Averaged Navier-Stokes (RANS) equations models, such as ADREA-HF, by their conceptual basis calculate ensemble average concentrations. 

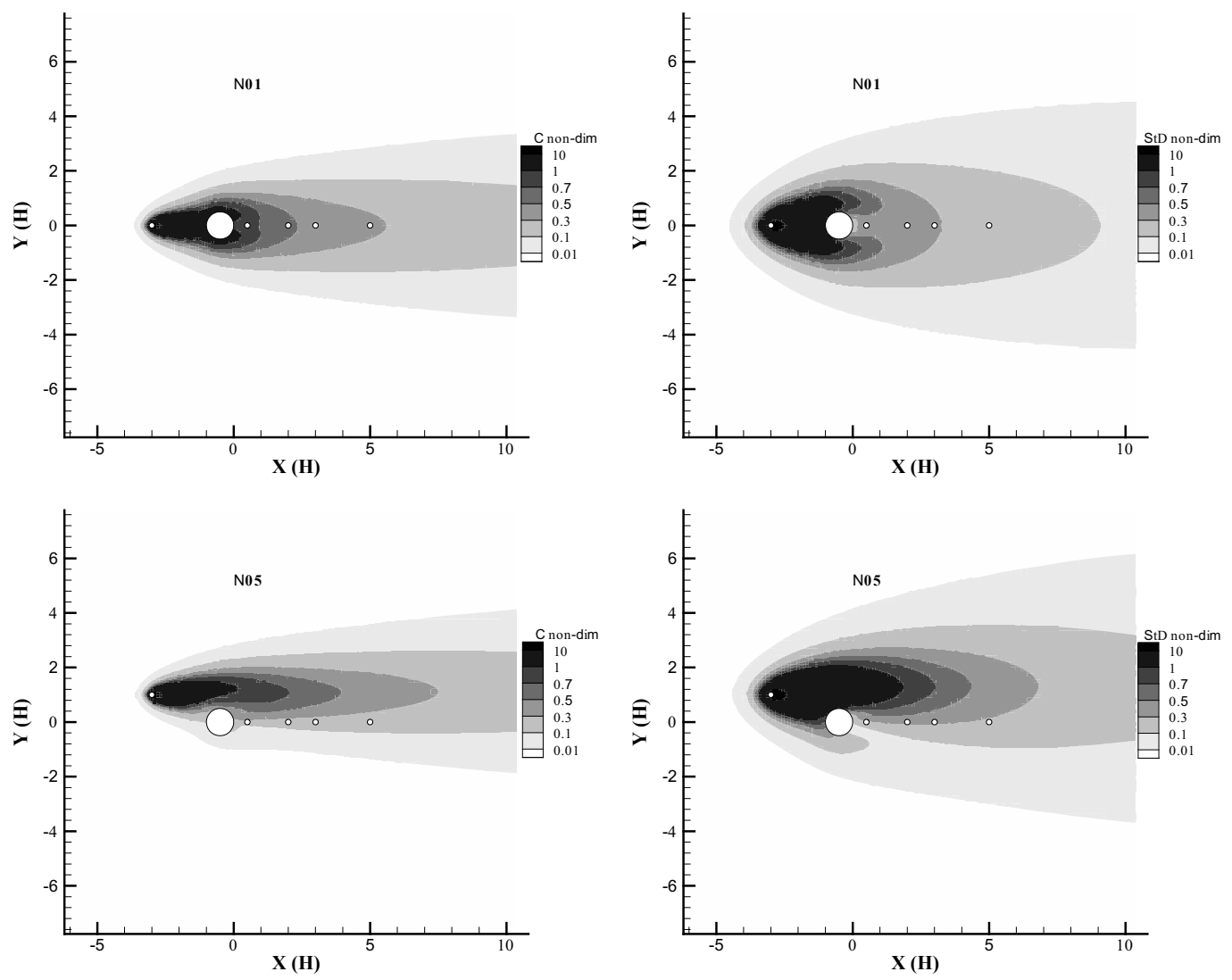

Figure 2. Contours of the non-dimensional calculated ammonia concentration and concentration fluctuations for cases N01 and N05, at the horizontal plane at the height of the gas source and detectors (both shown with circles); spatial dimensions are given in cylindrical obstacle diameter lengths $(H)$.

The comparisons are presented in Table 2 for the concentrations and the concentration fluctuations. The ratio of the calculated to the experimental value is set as the quantitative measure of the model performance. The agreement between the model results and the measured values is very good for the concentrations (ratio of calculated to experimental values equal to 0.9 on the average) with the modelled values slightly underpredicting the measurements. On the other hand the model-predicted concentration fluctuations overpedict the measured values by a factor slightly less than 2 .

As one experiment was conducted for each ammonia source location, it is not possible to apply the same averaging methodology as for the experiments involving propane. Along-wind profiles of computed ammonia concentrations and concentration fluctuations downwind of the obstacle, together with the field measurements for comparison purposes, are shown in Figure 3. The profiles are drawn on the axis defined by the centre of the obstacle and the ideal wind direction (at the height of the gas detectors). Ys denotes the distance of the gas source from the building centreline in the crosswind direction. For small values of Ys (N02), concentrations and concentration fluctuations are of the same order of magnitude and the model-calculated values compare rather well with the measurements. For larger lateral displacements of the source (N11) concentration fluctuations are larger than average concentrations, and the model predictions in general overestimate the measurements.

Table 2. Comparison of measured and calculated propane concentrations and concentration fluctuations at distances $0.5 \mathrm{H}$ and $3 \mathrm{H}$ downwind of the obstacle.

\begin{tabular}{|c|c|c|c|c|c|c|c|c|}
\hline & \multicolumn{4}{|c|}{ Concentration (non-dim.) } & \multicolumn{3}{c|}{ Concentration Fluctuation (non-dim.) } \\
\hline & \multicolumn{2}{|c|}{ Measured } & \multicolumn{2}{c|}{ Calculated } & \multicolumn{2}{c|}{ Experimental } & \multicolumn{2}{c|}{ Calculated } \\
\hline & $\mathbf{0 . 5 H}$ & $\mathbf{3 . 0 H}$ & $\mathbf{0 . 5 H}$ & $\mathbf{3 . 0 H}$ & $\mathbf{0 . 5 H}$ & $\mathbf{3 . 0 H}$ & $\mathbf{0 . 5 H}$ & $\mathbf{3 . 0 H}$ \\
\hline N01 & 0.423 & 0.238 & 0.262 & 0.147 & 0.537 & 0.278 & 0.765 & 0.425 \\
\hline N02 & 0.319 & 0.171 & 0.295 & 0.168 & 0.478 & 0.235 & 0.908 & 0.512 \\
\hline N05 & 0.288 & 0.175 & 0.280 & 0.158 & 0.416 & 0.236 & 0.845 & 0.475 \\
\hline N11 & 0.338 & 0.238 & 0.288 & 0.164 & 0.372 & 0.271 & 0.899 & 0.510 \\
\hline N07 & 0.204 & 0.122 & 0.276 & 0.156 & 0.339 & 0.199 & 0.829 & 0.465 \\
\hline Average & 0.314 & 0.189 & 0.280 & 0.158 & 0.429 & 0.244 & 0.849 & 0.478 \\
\hline Calc/Exper & & & 0.89 & 0.84 & & & 1.98 & 1.96 \\
\hline
\end{tabular}



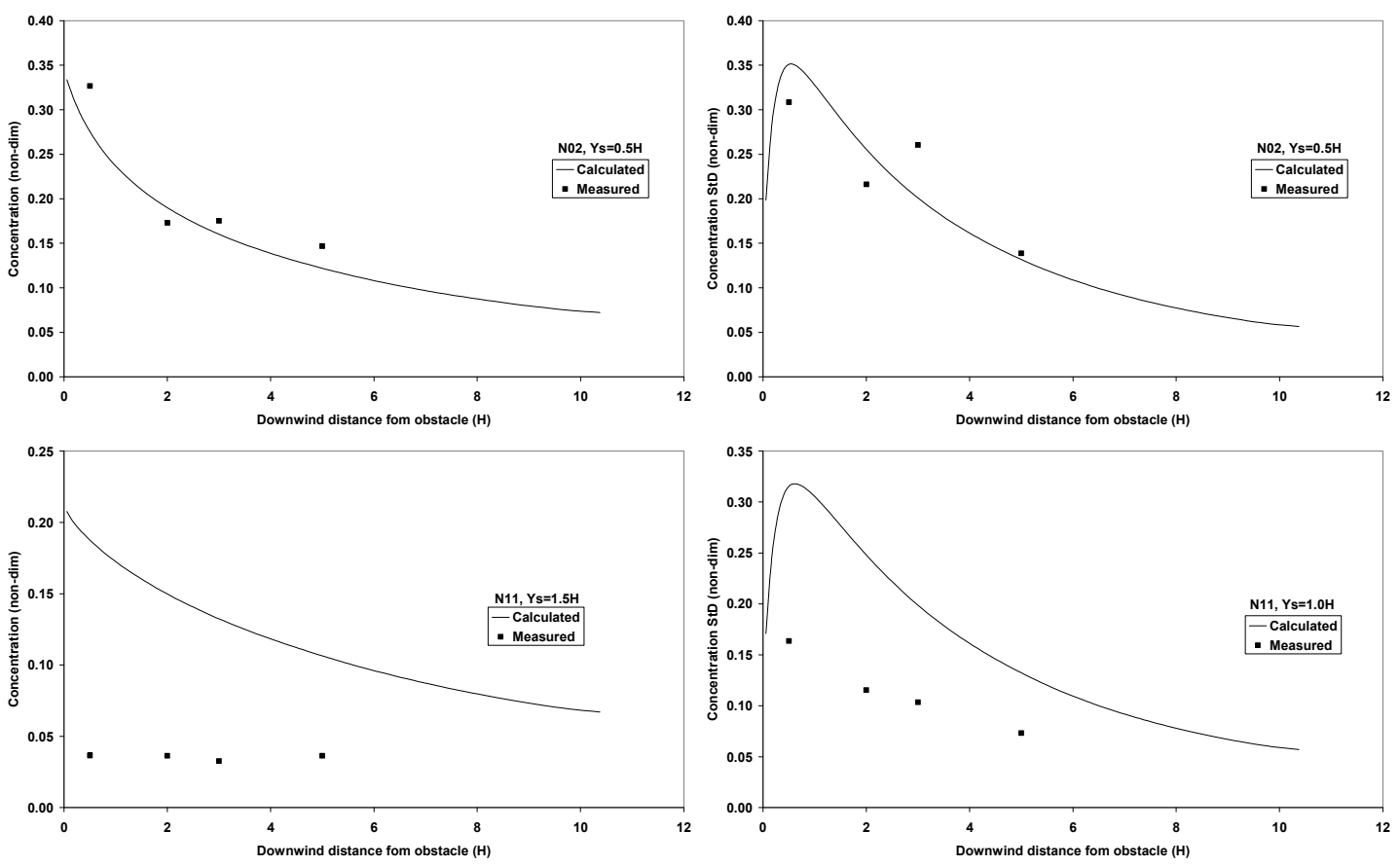

Figure 3. Comparison of computed ammonia concentration and concentration fluctuation profiles with field experimental data downwind the obstacle, for two different positions of the gas source.

Figure 4 presents a comparison between dispersion around a cubical obstacle (presented in Mavroidis et al., 2007) and dispersion around a cylindrical obstacle (with the same dimensions) presented in the current paper. The measured and model-calculated ammonia concentrations and concentration fluctuations at the sensor located at $0.5 \mathrm{H}$ downwind of the obstacles are plotted for the different lateral displacements of the sources. It is noted that for the cube case the concentrations decrease as the source is displaced laterally more abruptly than for the cylinder. Concentrations are also higher in the cube case when the source is at or near the centreline. Concentration fluctuations for the cube case decrease as the source is displaced laterally, while for the cylinder they present a peak value when the source is placed a $1 \mathrm{H}$ distance from the centreline. The model-calculated values overpredict the measurements at most locations, except for the fluctuations peak mentioned above.

\section{CONCLUSIONS}

Computational simulations of atmospheric dispersion around an isolated cylindrical model building have been presented in this paper. The relevant field experiments involved two gas sources (ammonia and propane) placed upwind of the obstacle and gas detectors downwind of the obstacle, along the wind direction. The propane source was always located on the cylinder's plane of symmetry defined by the wind direction. The ammonia source was displaced in each experimental trial to a different lateral position. Average concentrations and concentrations standard deviation (fluctuations) have been calculated using the CFD model ADREA-HF.

The simulations have revealed several characteristic dispersion features, such as the bifurcation of the plume for a centrally placed source, or the entrainment of the plume in the building's wake for a laterally displaced source. Experimental and calculated ensemble-averaged propane concentrations and concentration fluctuations have been used for model evaluation purposes. Concentrations are predicted rather well by the model, while fluctuations are overpredicted by a factor of 2 . Along-wind direction profiles downwind of the buildings show that concentrations and concentration fluctuations are of the same order of magnitude for a source placed near the centreline and are predicted well by the model. For a laterally displaced source, fluctuations are higher than average concentrations and both are overpredicted by the model.

Comparisons of experimental and model results for dispersion around a cubical and a cylindrical obstacle show that for the cube case the concentrations decrease as the source is displaced laterally more abruptly than for the cylinder. Concentrations are also higher in the cube case when the source is at or near the centreline. Concentration fluctuations for the cube case decrease as the source is displaced laterally, while for the cylinder they present a peak value when the source is placed a $1 \mathrm{H}$ distance from the centreline. The model-calculated values overpredict the measurements at most locations, except for the fluctuations peak mentioned above. 
Future work will include more complete and in depth examination of the issues mentioned in this short paper, as well as the investigation of parameters such as turbulence model, wind direction fluctuations and variability in the field experiments.
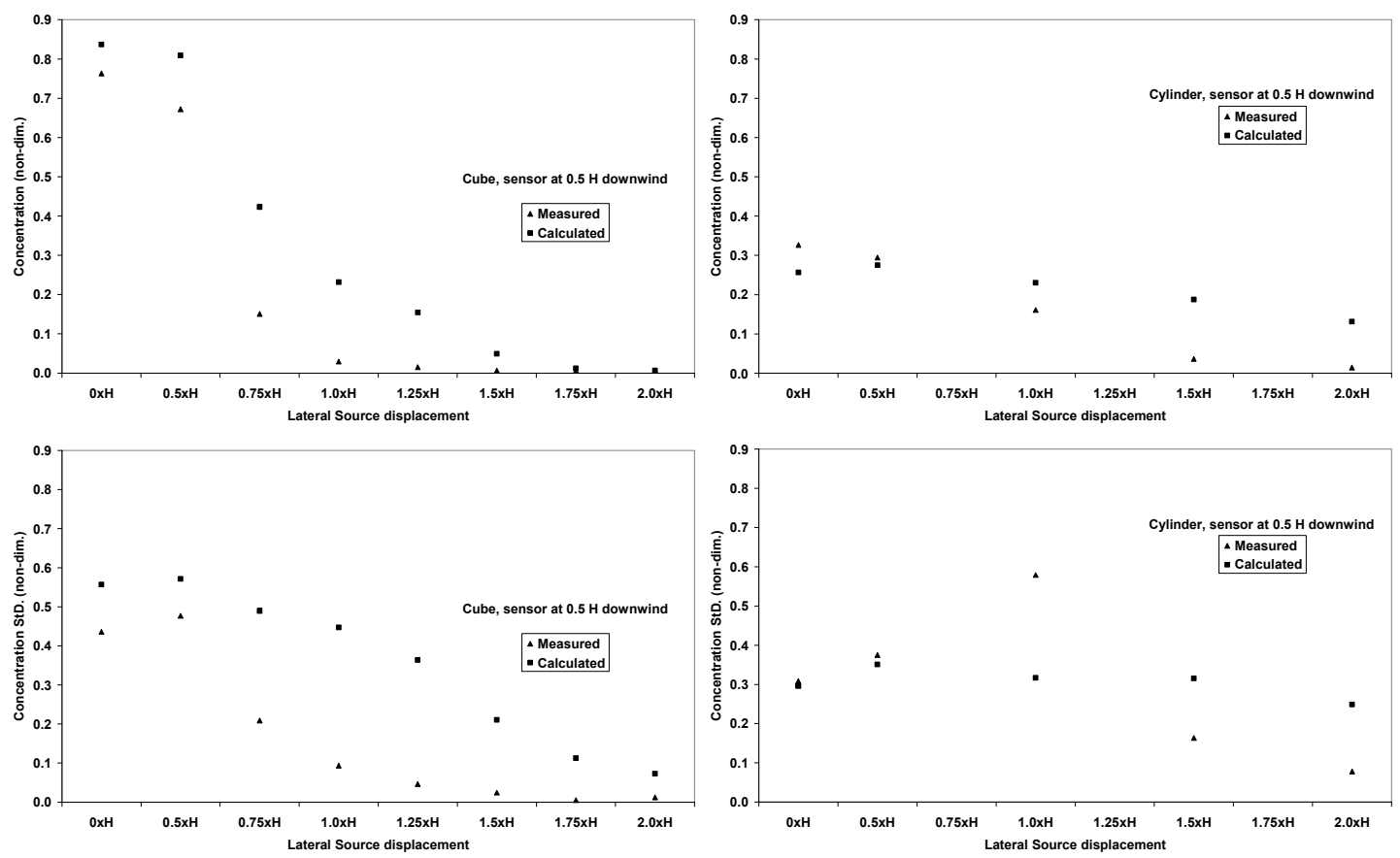

Figure 4. Comparison between dispersion around a cubical and a cylindrical obstacle: measured and model-calculated ammonia concentrations and concentration fluctuations for the different lateral source displacements

Acknowledgements: The field experiments simulated here were conducted with the support of the Atmospheric Dispersion Modelling Group at DSTL Porton Down, under agreement 2044/15/CBDE.

\section{REFERENCES}

Andronopoulos, S., D. Grigoriadis, A. Robins, A. Venetsanos, S. Rafailidis and J.G. Bartzis, 2002: Threedimensional modelling of concentration fluctuations in complicated geometry. Environmental Fluid Mechanics, 1, 415-440.

Mavroidis, I., R.F. Griffiths and D.J. Hall, 2003: Field and wind tunnel investigations of plume dispersion around single surface obstacles. Atmospheric Environment, 37(21), 2903-2918.

Mavroidis, I., S. Andronopoulos, J.G. Bartzis and R.F. Griffiths, 2007: Atmospheric dispersion in the presence of a 3dimensional cubical obstacle: modelling of mean concentration and concentration fluctuations. Atmospheric Environment, 41, 2740-2756. 